\title{
Sampling Frequency and the Performance of different Types of Technical Trading Rules
}

\author{
Robert Hudson, Grank McGroarty and Andrew J. Urquart
}

\begin{abstract}
The predictive ability of technical trading rules has been studied in great detail however many papers group all technical trading rules together into one basket. We argue that there are two main types of technical trading rules, namely rules based on trend-following and mean reversion. Utilising high-frequency commodity ETF data, we show that mean-reversion based rules perform increasingly better as sampling frequencies increase and that conversely the performance of trend-following rules deteriorate at higher-frequencies. These findings are possibly related to noise created by high-frequency traders.
\end{abstract}

Keywords: Technical Analysis; High-Frequency Trading; Commodity ETFs; Market Efficiency

JEL classification: G10; G11; G12; G14 


\section{Introduction}

Technical analysis is the use of past market data to forecast the direction of future prices and is widely employed by practitioners, with Menkhoff (2010) showing that $87 \%$ of fund managers place some importance on technical analysis when making investment decisions. The seminal paper by Brock et al (1992) shows that the simple moving average rule and trade range break rule both have significant predictive power on daily DJIA data from 1897 to 1986. Similar results have been found in other markets thus suggesting that technical analysis has strong predictive power. ${ }^{1}$ The majority of studies have examined the profitability of technical trading rules at daily frequency, however as Neely and Weller (2003) note, most technical traders transact at high-frequency. Also, Kirilenko and Lo (2013) point out that the majority of trades by volume on U.S. exchanges across all financial markets are high-frequency transactions and so high-frequency technical trading must be a popular tool for investors. The literature on high-frequency technical trading generally suggests that there is little profitability from technical trading at high frequency (for instance Neely and Weller 2003; Marshall et al 2008; Frömmel and Lampaert 2016), although Narayan et al (2015) do show significant profits from trading Brazilian, Indian and South African exchange rates on 5 minute frequency data.

The vast majority of studies examine a 'universe' of technical trading rules and show average results across rules. However there are two main types of technical trading rule, namely rules based on trend-following rules and those based on mean-reversion. Trend-following rules follow the old adage that 'the trend is your friend' and attempt to identify and follow trends until they change direction. In contrast, mean-reversion rules aim to identify overbought (or oversold) stocks and excess optimism (pessimism), and therefore go against the trend. The two types of rules are based on fundamentally opposite reasoning and so failing to separate them when examining the performance of technical trading rules is likely to lead to spurious conclusions.

In this paper, we examine the performance of a selection of trend following and mean-reversion technical trading rules in the high-frequency context. We employ a range of parameters for each trading rule on commodity ETF markets sampled at 5 minute intervals and find that trendfollowing rules are more successful at lower frequencies and mean-reversion rules are more successful at higher frequencies, which could be due to the noise of HFTs at high-frequency. Therefore, there is a need to separate these two types of technical trading rules whenever examining a group of technical trading rules.

\section{Methodology and Data}

We study a range of technical trading rules since Shynkevich (2012) notes, it is critical to construct an appropriate universe of technical trading rules because it may directly influence the results since too few rules may lead to biases in statistical inference due to data mining while loading the sample with too many rules may also cause a bias by reducing the power of the statistical tests. Therefore we study 6 trend-following rules and 2 mean-reversion rules. The trend-following rules are the simple moving average (SMA) rules, exponentially-weighted moving average (EWMA), weighted moving average (WMA) rule, the Aroon indicator, the Filter rule and the Channel Breakout rule. The two mean-reversion rules we employ are Bollinger bands (BBs) and the relative strength indicator (RSI), which both attempt to identify

\footnotetext{
${ }^{1}$ For instance see Hudson et al 1996; Fifield et al 2008; Hsu et al 2016.
} 
overbought or oversold stocks. We employ a range of parameters in order to capture the full dynamics of each trading rule and in total we study 9,736 different technical trading rules. More information about these trading rules and the parameter specification are presented in the online appendix. Regarding performance criteria, we study the daily mean excess return ${ }^{2}$, which are the daily returns minus the risk-free rate, Sharpe ratio, Sortino ratio and breakeven transaction costs.

We collect 5-minute transaction data from Thomson Reuters tick history and we focus our analysis on PowerShares commodity ETFs. Commodity ETFs have increased in popularity recently and offer investors a simple way to gain exposure to commodities, which are thought of as an asset class suitable for diversification in investment portfolios and as a hedge against economic downturns. We study the 4 most liquid commodity ETFs issued by PowerShares which consist of PowerShares DB Agriculture Fund (DBA), PowerShares DB Base Metals Fund (DBB), PowerShares DB Commodity Index Tracking Fund (DBC), and PowerShares DB Oil Fund (BDO) $)^{3}$.

We study the 5-year period from $4^{\text {th }}$ January 2010 to $31^{\text {st }}$ December 2015 and for maximum liquidity, only the core trading session of the NYSE from $9.30 \mathrm{am}$ to $4.00 \mathrm{pm}$ is considered. We follow Alsayed and McGroarty (2014) and remove data which is problematic according to the following criteria:

1. bid price $\geq$ ask price;

2. bid volume $=0$ and/or ask volume $=0$

3. ask price $>$ bid price by more than $25 \%$

We resample the 5-minute data to form 10-minute, 15-minute, 30-minute and 60-minute samples and calculate logarithmic returns. The descriptive statistics are reported in Table 1 and show that each ETF has a negative mean and that the Ljung-Box statistic becomes larger as the data frequency increases suggested increased autocorrelation at higher frequencies.

Table 1: Descriptive statistics of each commodity ETF returns. JB refers to the Jarque-Bera p-value while $Q(n)$ refers to the Ljung-Box statistics.

\footnotetext{
${ }^{2}$ Daily

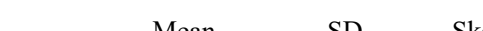

Panel A: 5-minute

${ }^{3}$ More DBB -0.000075

https:/ DBC -0.000054

BDO -0.000105

Panel B: 10-min

DBA $\quad-0.0004$

DBB $\quad-0.00014$

SD Skew

$0.001474-0.08$

$0.002128 \quad 0.030$

$0.004174-0.030$

$0.002237 \quad-1.09$

$0.001693-0.06$

DBC -0.00010

$0.002567-0.26$

$\begin{array}{ccc}0.001474 & -0.08 \\ 0.002128 & 0.030 \\ 0.004174 & -0.030 \\ 0.002237 & -1.09 \\ & \\ & 0.001693 & 0.06 \\ 0.002567 & -0.26 \\ 0.001582 & -1.15 \\ 0.002831 & \\ & & \\ & 0.002123 & 0.13\end{array}$

Kurt JB

$Q(6)$

$Q(12)$

$$
Q(24)
$$

Ich's

$4849.82 \quad 0.00$

$622.51 \quad 0.00$

$50369.57 \quad 0.00$

$525.94 \quad 0.00$

3751.59

$59 \quad 0.00$

66.19

0.00

$11841 * * *$
$2876.1 * * *$

$2876.1 * * *$
$25607 * * *$

$1918.2 * * *$

$11845^{* * *}$

$2965.8 * * *$

$25608 * * *$

$2061.74 * * *$

$308.64 * * *$

$241.46 * * *$

$302 * * *$

$253.00 * * *$

$13790 * * *$

$3197.4 * * *$

$20608 * * *$

$2113.95 * * *$

Panel C: 15-min

DBA $\quad-0.000066$ 


\section{Empirical Results}

Table 2 reports the summary results for our trend following strategies at various frequencies. We report the mean results from the trend-following strategies and the mean-reversion strategies due to space constraints but all of the individual trend-following and mean-reversion rules give qualitatively similar results. ${ }^{4}$ We can see that the mean buy (sell) returns of the trend-following rules are all negative (positive) and therefore the mean buy-sell are all negative. Correspondingly, all Sharpe ratios, Sortino ratios and breakeven transaction costs are negative. Moreover, as the frequency of the data sampling increases from 60-minute to 5minute, the performance of the rules deteriorates. Conversely, we find that mean-reversion strategies do better at higher frequencies, with significant positive mean buy-sell returns for mean-reversion rules at the 5-minute frequency. As we move to lower frequency data, the performance of the mean-reversion rules fall and the mean excess returns actually turn negative at frequencies of 30-minutes and lower.

Table 2: The technical trading results. TF and MR refer to the trend-following and mean-reversion strategies respectively. $\mathrm{N}$ (Buys) and $\mathrm{N}$ (Sells) refer to the number of buy and sell signals, while Buy and Sell refer to the mean return generated from a buy and sell signal. Buy-sell and t-stat are the mean period buy return minus the mean sell returns and the corresponding t-statistics. Excess returns denotes the daily excess returns, while Sharpe and Sortino refer to the Sharpe and Sortino ratios. BTCs refers to the breakeven transaction costs.

5-min N(Buys) N(Sells) Buy Sell Buy-Sell t-stat Excess Returns Sharpe Sortino BTCs

Panel A: 5-minute

C2018, Elsevięp This manuscript version is made available under the CC-BY-NC-ND 4.0 license http://creativecommons.org/licenses/by-nc-nd/4.0/

$\begin{array}{llllllllll}19851 & 20916 & -0.003708 & 0.002927 & -0.006423 & -1.73 & -0.000706 & -0.5215 & -0.2302 & 0.0035 \\ & 22698 & 22253 & 0.000557 & -001766 & 0.002324 & 3.88 & 0.001341 & 0.7972 & 0.8884\end{array}$

$\begin{array}{llllllllllll}M R & 22698 & 22253 & 0.000557 & -0.001766 & 0.002324 & 3.88 & 0.001341 & 0.7972 & 0.8884 & 0.0004\end{array}$




\section{Conclusion}

This paper suggests that investors and academics alike should distinguish between the two main types of technical trading rules when trading. By studying highly liquid commodity ETF markets, we show that trend-following technical trading rules offer no predictive power to investors at any frequency but that mean-reversion rules offer significant profits at the 5-minute frequency. As the sampling frequency decreases, the profits from mean-reversion rules falls indicating that investors should implement mean-reversion rules at the higher frequencies and not trend-following rules. One might speculate that at high frequencies deviations from fair value (with no arbitrage opportunities available) can occur., perhaps attributable to the noise generated by very high-frequency trade, However, these deviations are swiftly eliminated by the sort of informed traders proposed in the seminal model of Grossman and Stiglitz (1980) resulting in mean-reverting behavior at high frequencies but more random movements at lowerfrequencies. 


\section{References}

Alsayed, H., McGroarty, F. (2014). Ultra-High-Frequency Algorithmic Arbitrage Across International Index Futures. Journal of Forecasting, 33, 391-408.

Brock, W., Lakonishok, J. and LeBaron, B. (1992). Simple Technical Trading Rules and the Stochastic Properties of Stock Returns. Journal of Finance, 47(5), 1731-1764.

Fifield, S.G.M., Power, D.M. and Sinclair (2005). An analysis of trading strategies in eleven European stock markets. The European Journal of Finance, 11(6), 531-548.

Frömmel, M., Lampaert, K. (2016). Does frequency matter for intraday technical trading? Finance Research Letters, 18, 177-183.

Grossman, S. J., \& Stiglitz, J. E. (1980). On the impossibility of informationally efficient markets. The American Economic Review, 70(3), 393-408.

Hsu, P-H., Taylor, M. P., Wang, Z. (2016). Technical trading: Is it still beating the foreign exchange market? Journal of International Economics, 102, 188-208.

Hudson, R., Dempsey, M. and Keasey, K. (1996). A note on the weak form efficiency of capital markets: The application of simple technical trading rules to UK stock prices - 1935 to 1994. Journal of Banking \& Finance, 20(6), 1121-1132.

Kirilenko, A., A., Lo, A. W. (2013). Moore's Law versus Murphy's Law: Algorithmic Trading and Its Discontents. Journal of Economics Perspectives, 27(2), 51-72.

Marshall, B. R., Cahan, R. H., Cahan, J. M. (2008). Does intraday technical analysis in the U.S. equity market have value? Journal of Empirical Finance, 15, 199-210.

Menkhoff, L. (2010). The use of technical analysis by fund managers: International evidence. Journal of Banking and Finance, 34(11), 2573-2586.

Narayan, P. K., Mishra, S., Narayan, S., Thurasisamy, K. (2015). Is Exchange Rate Trading Profitable? Journal of Financial Markets, Institutions and Money, 38, 217-229.

Neely, C. J., Weller, P. A. (2003). Intraday technical trading in the foreign exchange market. Journal of International Money and Finance, 22, 223-237.

Shynkevich, A. (2012). Short-term predictability of equity returns along two style dimensions. Journal of Empirical Finance, 19, 675-685. 